\title{
Magnetic Resonance Cholangio-Pancreatography in Patients with Acute Cholecystitis and Cholestatic Liver Pattern - What to Expect?
}

\author{
Ali Al Orf ${ }^{1}$, Khawaja Bilal Waheed², Ali Salman Alshehri³ ${ }^{3}$, Mushref Ali Algarniं, Bilal Altaf ${ }^{5}$, \\ Muhammad Amjad 6 , Ayman Abdullah Alhumaid7 ${ }^{7}$ Zechariah Jebakumar Arulanantham ${ }^{8}$
}

\begin{abstract}
${ }^{1}$ Department of Radiology, King Fahad Military Medical Complex, Dhahran, Saudi Arabia. ${ }^{2}$ Department of Radiology, King Fahad Military Medical Complex, Dhahran, Saudi Arabia. ${ }^{3}$ Department of Radiology, King Fahad Military Medical Complex, Dhahran, Saudi Arabia. ${ }^{4}$ Department of Radiology, King Fahad Military Medical Complex, Dhahran, Saudi Arabia. ${ }^{5}$ Department of General Surgery, King Fahad Military Medical Complex, Dhahran, Saudi Arabia. ${ }^{6}$ Department of Internal Medicine, King Fahad Military Medical Complex, Dhahran, Saudi Arabia. ${ }^{7}$ Department of Radiology, King Fahad Military Medical Complex, Dhahran, Saudi Arabia. ${ }^{8}$ Prince Sultan Military College of Health Sciences, Dhahran, Saudi Arabia.
\end{abstract}

\section{ABSTRACT}

\section{BACKGROUND}

Acute cholecystitis is a potentially serious condition and usually needs to be treated in the hospital. Identification of a common bile duct (CBD) stone before cholecystectomy is of concern for the treating physicians as management may change. Magnetic Resonance Cholangiopancreatography (MRCP) can help in identifying causes of biliary obstruction (if present) and adequately delineate biliary tree in selected patients with limited or abnormal ultrasounds and cholestatic liver pattern. Therefore, we aim to demonstrate imaging findings of MRCP in such patients of acute cholecystitis, and highlight the diagnostic ability of MRCP in biliary ductal evaluation as well.

\section{METHODS}

This secondary data analysis from hospital records was performed in Radiology department at our Hospital in Dhahran from August 2017 to 2019. All clinically suspected and ultrasound supported cases of acute cholecystitis who were referred for MRCP studies were included. Dilated CBDs (more than $4 \mathrm{~mm}$ in caliber) with partial visualization or non-discernible causes of CBD dilatations, rising or persistently raised LFTs (denoting cholestatic pattern) were the common indications for the MRCP referrals. Patients with chronic cholecystitis, previous hepatobiliary surgery, pregnant patients, and those contraindicated to MRI were excluded.

\section{RESULTS}

Of the 104 patients, majority (60\%) were females. The mean age was 43 years. Twothirds of patients were having normal CBDs (68.3\%), while nearly one-third (31.7\%) had dilated CBDs, and half of these (16.4\%) showed identifiable causes of obstruction that were later confirmed on ERCP and histopathology. Thirteen patients (12.5\%) had associated anomalies. Sensitivity and specificity of MRCP in CBD evaluation were measured as $90.5 \%$ (CI, 79.3-96.8) and $86.2 \%$ (CI, 73.7-94.3) respectively. The length of the hospital stay was found to be significantly less in laparoscopic cases compared to open cholecystectomies $(\mathrm{P}=0.0005)$.

\section{CONCLUSIONS}

Magnetic resonance cholangiopancreatography can help in identifying the causes and anomalies in patients with acute cholecystitis having deranged or obstructive liver function.

\section{KEY WORDS}

Cholecystitis, Common Bile Duct, Magnetic Resonance Cholangiopancreatography
Corresponding Author: Khawaja Bilal Waheed, Consultant General Radiologist, King Fahad Military Medical Complex, Dhahran, Saudi Arabia.

E-mail: khawaja@kfmmc.med.sa DOI: 10.14260/jemds/2020/530

How to Cite This Article:

Orf $A A$, Waheed $K B$, Alshehri $A S$, et al. Magnetic resonance cholangiopancreatography in patients with acute cholecystitis and cholestatic liver patternwhat to expect? J Evolution Med Dent Sci 2020;9(34):2436-2441, $10.14260 / \mathrm{jemds} / 2020 / 530$

Submission 13-05-2020, Peer Review 07-07-2020, Acceptance 14-07-2020, Published 24-08-2020.

Copyright (C) 2020 JEMDS. This is an open access article distributed under Creative Commons Attribution License [Attribution 4.0 International (CC BY 4.0)] 


\section{BACKGROUND}

Acute cholecystitis is a potentially serious condition and usually needs to be treated in the hospital.[1] If the diagnosis is confirmed, early surgery is indicated. Acute cholecystitis can be classified into calculous cholecystitis (with gallstones) and acalculous cholecystitis (without gallstones). Gallstones cause blockage to the flow of bile and account for $90 \%$ of cases. Acute calculous cholecystitis is caused by an obstruction to cystic duct, leading to distention of the gallbladder compromising its blood flow and lymphatic drainage and causing mucosal ischemia and necrosis. Acalculous cholecystitis can occur by accidental damage to the gallbladder during major surgery, serious injuries or burns, sepsis (systemic infection), severe malnutrition or AIDS (Acquired Immunodeficiency Syndrome). Accurate and timely diagnosis is important to initiate adequate management.[2,3] The condition can be life threatening and may require surgery. ${ }^{[4]}$

In patients with acute cholecystitis, identification of a common bile duct (CBD) stone before cholecystectomy is of concern for surgeons, gastroenterologists, and radiologists, particularly if the liver function tests are found to be deranged or patients have an obstructive liver pattern on blood tests. Ultrasound is the preferred imaging modality for the evaluation of clinically suspected acute cholecystitis, ${ }^{[5]}$ and to exclude its mimics.[6] In patients with calcular cholecystitis, presence of a CBD stone (choledocholithiasis) or dilatation may guide clinicians to adopt a different approach for patient management. For example, they may opt surgery in case of no CBD stone/dilatation or they may proceed with an initial ERCP (Endoscopic Retrograde Cholangio-pancreatography) in case of stone or other cause of obstruction. Although ultrasound may be able to detect a dilated or obstructed CBD, it may also detect a stone or intraluminal debris or sludge in some cases. However, it remains limited in delineating the entire length of the CBD mostly due to obscuration by bowel gases. Fatty or large-built patients may cause further limitations in assessment, and so are the irritable or uncooperative patients (not holding breath adequately during the scanning). In addition, ultrasound is operator dependent and requires both expertise and experience to evaluate difficult or challenging cases.

Magnetic resonance cholangiopancreatography (MRCP) can accurately detect choledocholithiasis in patients with acute cholecystitis.[7] Its multiplanar cross-sectional imaging and exquisite tissue characterization have greatly benefitted such patients for whom ultrasounds remain inconclusive or limited for CBD evaluation. Time, cost, claustrophobia, and certain contraindications to MR magnet are few of its limitations. Patient's cooperation (to avoid movement or breathing artefacts), and at times intravenous contrast administration may be needed to outline any mass (cholangiocarcinoma, periampullary or pancreatic head carcinoma) or for differentiation of certain imaging findings. MRCP is a non-invasive and safe option, ${ }^{[8]}$ when compared to ERCP for imaging biliary tree and biliary obstruction, ${ }^{[9]}$ and has largely replaced ERCP as the gold standard for diagnosis of choledocholithiasis and obstructive jaundice,[10] achieving nearly similar sensitivity (90-94\%) and specificity (95-99\%) without utilizing ionizing radiation, intravenous contrast, or the complications inherent to ERCP. It also helps to delineate any congenital biliary abnormality (e.g., choledochal cyst, low
CBD insertion), and to document any concomitant complications (e.g., acute pancreatitis, changes pertaining to cholangitis).[11] These additional information and visual anatomy of the biliary tree prior to surgery help clinicians to plan management and offer better patient counselling and help surgeons to adopt better operative approach.

Various studies in the literature have discussed the role of MRCP in diagnosis of biliary obstruction, ${ }^{[12]}$ and to document complications of acute cholecystitis.[13] However, imaging patterns of MRCP findings have not been categorically described. Therefore, we aim to highlight MRCP findings to demonstrate its role in such indicated patients.

\section{METHODS}

This secondary data analysis from hospital records was performed in the radiology department at our hospital in Dhahran from August 2017-2019, evaluating electronic health record of all clinically suspected and ultrasound supported cases of acute cholecystitis who were referred for MRCP studies ( $\mathrm{N}=104)$. Dilated CBDs (more than $4 \mathrm{~mm}$ in calibers) with partial visualization or non-discernible causes of CBD dilatations, rising or persistently raised LFTs (denoting cholestatic patterns) were seen common indications for the MRCP referrals. Elevation in alkaline phosphatase (ALP) and bilirubin in disproportion to ALT and AST was considered cholestatic pattern. Ultrasound findings of presence of gallstones in combination with the sonographic Murphy sign, gallbladder wall thickening ( $>3 \mathrm{~mm}$ ) and pericholecystic fluid, sludge or gallbladder distension were considered suggestive or supportive of acute cholecystitis in appropriate clinical setting of right upper quadrant pain (with nausea, vomiting or fever). Patients with chronic cholecystitis, post-surgery or intervention (biliary stenting) cases, pregnant patients, and those contraindicated to MRI were excluded.

The study analysis was approved from the institutional review board (IRB), and was conducted in accordance with the Helsinki Declaration. All clinical and radiologic information were kept strictly confidential. A literature review was performed using an electronic search (Google Scholar, PubMed). Demographic information regarding age and gender of all patients was collected. Clinical information and MRI findings were acquired through patients' clinical notes from Hospital Information System (HIS), Radiology Information System (RIS), and the Picture Archiving and Communication System (PACS). Information about clinical presentation and follow up were recorded from the clinical notes on HIS.

Routine MRCP studies were performed on a 1.5 Tesla scanner (General Electric/ GE, Optima 450 W GEM, 2013, Florence, South Carolina, USA). Intravenous contrast (gadolinium-based agent, Dotarem, $0.1 \mathrm{mmol} / \mathrm{kg}$; Guerbet, France) was used only in few cases who were having suspicion of mass or infection. Imaging sequences included Coronal Single Shot Fast Spine Echo (CSSFSE) with respiratory gating (thickness $5 \mathrm{~mm}$, spacing 1.0), Axial (Ax) 2D FIESTA (Fast Imaging Employing Steady-State Acquisition) FAT SAT with respiratory gating (thickness $5 \mathrm{~mm}$, spacing 1.0), Ax T2 Propeller with respiratory gating (thickness $5 \mathrm{~mm}$, spacing 1.0), 3D MRCP with respiratory gating (thickness $0.8 \mathrm{~mm}$, spacing 0.0), Coronal (cor) T2 CUBE (multiplanar 3D) with respiratory gating (thickness $1.6 \mathrm{~mm}$, spacing 0.0 ), and Cor T2 
(thin) with respiratory gating (thickness $2.0 \mathrm{~mm}$, spacing 0.2 ). If needed, Ax DWI with respiratory gating (thickness $5 \mathrm{~mm}$, spacing 1.0), and Ax 2D or 3D Dual Echo T1 breath-hold inphase and out-phase (thickness $5 \mathrm{~mm}$, spacing 1.0) were acquired.

MRCP findings were categorized as: - (i) With normal CBD, (ii) With dilated CBD and cause identifiable, (iii) with dilated CBD and cause not identifiable. Any associated biliary ductal variants or anomalies (like pancreas divisum, choledochal cyst or low insertion of CBD) were also documented. Imaging was interpreted by two general/ body radiologists (each having more than 7 years of experience), who were kept blinded of clinical information and final results, with substantial interobserver agreement (Cohen's Kappa- 0.81). In cases of minor differences, MRI findings were made by consensus reporting. MRCP findings (in cases of obstructive causes) were confirmed on subsequent ERCP (Endoscopic Retrograde Cholangiopancreatography) and histopathology. A normal follow up imaging with normalization of LFTs were considered confirmation for negative cases (sensitivity and specificity of MRCP were measured for CBD evaluation.). Operated cases were followed to determine length of hospital stay after open or laparoscopic procedures. The statistical analysis was carried out using Statistical Package for Social Sciences (SPSS, version 22). Chi-square test was used to determine association, and p-values less than 0.05 were considered significant.

\section{RESULTS}

Of the 104 patients, $60 \%(n=62)$ were females, and $40 \%$ $(n=42)$ were males. The mean age was 43 years (std. deviation16.8). Thirteen patients (12.5\%) were having associated anomalies. Acute cholecystitis patients (57 calculus, 5 acalculous cholecystitis) with normal CBDs, dilated CBDs with identifiable causes, and dilated CBDs without identifiable causes were found in $68.3 \%, 16.4 \%$ and $15.3 \%$ respectively.

Identifiable causes included CBD stones (choledocholithiasis, $n=9)$, choledochal cysts $(n=3)$, benign stricture ( $\mathrm{n}=2$ ) and masses (ampullary/ periampullary, 2) [Fig. 2]. These cases were subsequently confirmed on ERCP and histopathology. Recent stone passages were assumed in cases where CBD dilatations (mostly mild, 4-6 $\mathrm{mm}$ ) were not seen associated with any identifiable causes. These cases were confirmed clinically by normalization or improvement in both symptoms and cholestatic liver pattern on subsequent laboratory testing (within 2 weeks interval). Sensitivity, specificity, positive predictive value and negative predictive values of MRCP in CBD evaluation were measured as $90.5 \%$ (CI, 79.3-96.8), 86\% (CI, 73.7-94.3), 87.2\% (CI, 77.4-93.2), and $89.8 \%(79.1-95.3)$ respectively; with True positives $(n=48)$, False Positives $(n=7)$, False Negatives $(n=5)$ and True Negatives $(n=44)$. False positives results occurred mostly because of breathing artefacts (with adjacent vessels giving ghost shadows like stones), while presence of either debris/ sludge or early ampullary lesion not clearly seen in false negative patients.

Associated anomalies were not seen significantly associated ( $p$ - value $=.53$ ) with the imaging patterns [Table. 1] Forty patients underwent laparoscopic surgery, and 30 patients had open cholecystectomies. The length of the hospital stay was seen significantly less in laparoscopic cases $(\mathrm{p}=.0005)$.

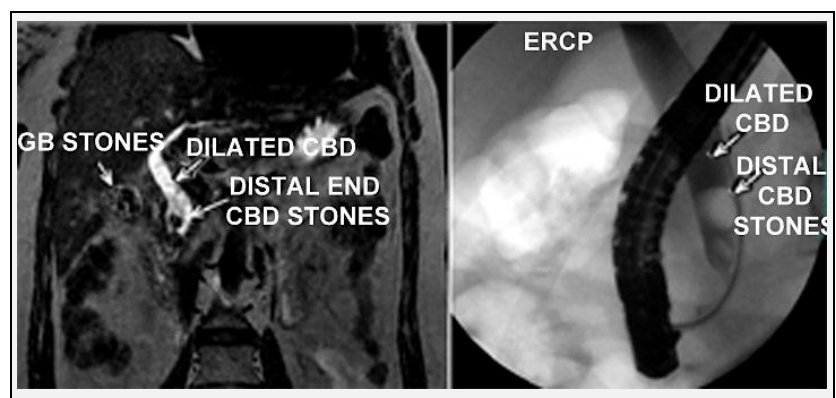

Figure 1. Selected coronal T2 reformat image (image on left) showing dilated CBD with two stones (and stones within the GB as well). Selected spot film fluoroscopic image during ERCP of same patient (image on right) showing filling defects corresponding to stones within the distal CBD.

GB-Gallbladder, CBD-Common Bile Duct, ERCP-Endoscopic Retrograde Cholangiopancreatography

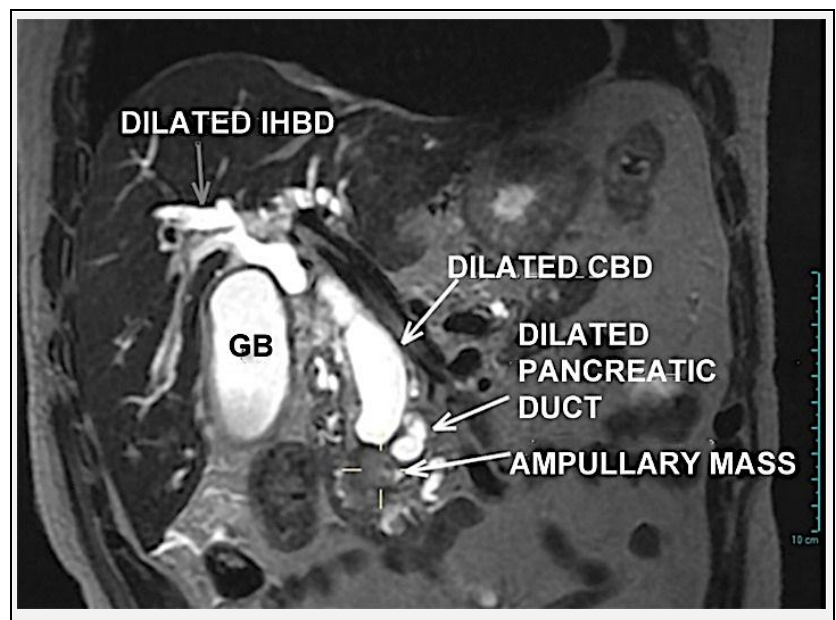

Figure 2. Coronal T2 reformat image of a patient showing an ampullary mass with dilated IHBD, GB, CBD and pancreatic duct. IHBD-Intra-Hepatic Biliary Duct, GB-Gallbladder, CBD-Common Bile Duct

\begin{tabular}{|c|c|c|c|c|}
\hline & \multirow{2}{*}{$\begin{array}{l}\text { Imaging } \\
\text { Findings }\end{array}$} & \multicolumn{2}{|c|}{ Associated Anomaly } & \multirow{2}{*}{ Total } \\
\hline & & Not Present & Present & \\
\hline \multirow{4}{*}{ MRCP } & A & $62(87.3 \%)$ & $9(12.7 \%)$ & $71(100.0 \%)$ \\
\hline & B & $16(94.1 \%)$ & $1(5.9 \%)$ & $17(100.0 \%)$ \\
\hline & \multirow{2}{*}{ Total ${ }^{\mathrm{C}}$} & $13(81.3 \%)$ & $3(18.8 \%)$ & $16(100.0 \%)$ \\
\hline & & 91 & 13 & 104 \\
\hline
\end{tabular}

\begin{tabular}{|cccc|}
\hline \multirow{2}{*}{ Surgery Type } & Less Than 3 Days & More Than 3 Days & Total \\
Laparoscopic & 31 & 9 & 40 \\
Open & 9 & 21 & 30 \\
Total & $\mathbf{4 0}$ & $\mathbf{3 0}$ & $\mathbf{7 0}$ \\
\hline Table 2. Distribution of Surgeries and Length of Stay \\
\hline \multicolumn{4}{|l}{} \\
\hline
\end{tabular}

Non-operated cases included either acalculous cholecystitis, debilitated non-surgical candidates (requiring cholecystostomy tube insertion), mild changes of cholecystitis (with suspicion of recent stone passage and subsequent improvement), and cancer patients (cholangiocarcinoma or peri-ampullary carcinoma that required either oncologic referral to nearby specialist hospital or underwent ERCP stenting). 


\section{DISCUSSION}

We found important implications of our study. Firstly, we observed that most of the clinically indicated MRCP patients were having normal or mildly dilated (no cause identifiable) CBD calibers. Therefore, it is emphasized that many of such patients can be kept under observation for monitoring of liver function tests despite having cholestatic liver function patterns or mildly dilated CBDs (on initial ultrasounds at presentation). Transient increase in liver function or cholestatic picture may result from either recent stone passage through CBD and compression on cystic duct or CBD by inflammatory process involving the gallbladder. Studies have shown that patients with normal and dilated (obstructed) CBD may show a different altered liver function tests with relatively variable threshold values. Chen JE and colleagues found that in patients with acute cholecystitis who had normal CBD diameter, normal or even mildly elevated bilirubin levels below a calculated threshold may obviate preoperative MRCP.[14] Their results showed a significant difference in the total and direct bilirubin levels of patients who had positive (1.94 vs $4.02 \mathrm{mg} / \mathrm{dL}$, respectively; $\mathrm{p}=0.013$ ) and negative ( $0.71 \mathrm{vs} 2.13 \mathrm{mg} / \mathrm{dL}$, respectively; $\mathrm{p}=0.02$ ) findings for CBD stone on MRCP. Chisholm PR et al demonstrated statistically significant odds ratios for transaminases $>3$ times the upper limit of normal, alkaline phosphatase above normal, lipase $>3$ times the upper limit of normal, total bilirubin $\geq 1.8 \mathrm{mg} / \mathrm{dL}$, and CBD diameter $>6 \mathrm{~mm}$, to predict choledocholithiasis.[15] Boys JA et al demonstrated that increasing CBD diameter of 6-9.9 $\mathrm{mm}$ were associated with $14 \%$ incidence of CBD stones, while $>10 \mathrm{~mm}$ with $39 \% .{ }^{[16]}$ Therefore, it is suggested that clinical assessment and laboratory parameters are important to foresee CBD stones or obstruction, and to refer patients for imaging.

Secondly, we observed stone to be the commonest cause of a dilated CBD (i.e., choledocholithiasis) associated with acute calculus cholecystitis. Choledocholithiasis with acute cholecystitis has always been an area of interest for the surgeons and may even be important for patient's management as presence of CBD stones may warrant an initial endoscopic retrograde cholangiopancreatography (ERCP) to remove CBD stones. Qiu Y et al suggested routine preoperative MRCP for diagnosis of associated choledocholithiasis prior to cholecystectomy for patients with gallstones is important for the surgical decision and treatment efficacy.[7] However, AlJiffry BO et al found that a direct laparoscopic cholecystectomy in patients with deranged liver function and normal CBD ultrasound avoided nearly $42 \%$ of unnecessary MRCPs.[17] We believe that incident dilated CBD in patients with acute cholecystitis may be strategic and sometimes justified to channel patients either to GB surgery (if no cause of obstruction identifiable, and recent passage of stone is suspected clinically and on laboratory test) or ERCP (if stones or mass detected) prior to GB or other surgery. Although not seen frequently on ultrasound (due to obscuration by bowel), presence of a double-duct sign (i.e., dilated CBD and pancreatic duct) can be sign of tumour. Sinha R and his colleagues found a $48 \%$ incidence of malignancy among patients with this specific sign, particularly in jaundiced patients.[18] Scanning time may be reduced for such patients by adopting limited sequences. Although we did not include pregnant patients with acute cholecystitis, however, this subset also appears to benefit from MRCP (if indicated) considering non-ionizing modality.[19] Kang SK et demonstrated that in hospitalized patients with suspected choledocholithiasis, a non-contrast abdominal MRI with HASTE (Half-Fourier Single-Shot Turbo Spin Echo) was like contrast-enhanced MRI with 3D-MRCP, offering potential for decreased scanning time and improved patient tolerability.[20]

Third important aspect of our study was observation of associated congenital biliary anomalies that were nicely demonstrated by MRCP in these patients. Although not statistically significant, these findings might be clinically relevant, as presence and documentation of such anomalies not only help to adopt a more careful approach during surgery but also for patients' counselling and prognosis. For example, presence of choledochal cyst and choledochocele on MRCP may help surgeons to better orientate patients about their problems and approach. Also, identification of a low-lying CBD or variant of CBD insertion may guide surgeons to adopt a more careful operative approach while ligating cystic duct, avoiding any biliary ductal damage or leak afterwards. Nasr MM presented safe surgical technique to minimize dissection and risk of injury related to the traditional laparoscopic cholecystectomy.[3] Length of hospital stay was seen significantly less in laparoscopic (30 patients) than with open (40 patients) cholecystectomies $(\mathrm{p}=.0005)$. Laparoscopy has now become the first-line approach to perform cholecystectomy in patients with acute cholecystitis.[21] Although for milder disease a conservative management might be an option. Loozen CS noted that cconservative treatment of acute calculous cholecystitis during index admission seemed feasible and safe, especially in patients with mild disease.[22] Barreiro Alonso $\mathrm{E}$ et al showed that not performing a cholecystectomy within two weeks after a first episode of mild acute biliary oedematous pancreatitis or cholecystitis contributed to patient readmission due to recurrent pancreatitis, resulting in avoidable treatment costs.[23] A recent study presented by Fleming CA et al showed that almost $90 \%$ of 157 patients with acute cholecystitis who were managed with a percutaneous cholecystostomy tube (PCT) recovered uneventfully without recurrent sepsis following PCT removal- a viable option for older, comorbid patients who were unfit for surgical intervention and was not associated with significantly increased mortality.[24] Hajibandeh $\mathrm{S}$ et al found that extended postoperative antibiotic therapy did not improve postoperative infectious or noninfectious outcomes in patients with mild or moderate acute calculous cholecystitis undergoing emergency cholecystectomy.[25]

One patient in our study had border-line wall thickening on ultrasound without identifiable GB stones. A Hepatobiliary Iminodiacetic Acid/ HIDA scan (also called cholescintigraphy, hepatobiliary scintigraphy or hepatobiliary scan) was therefore performed that suggested acute cholecystitis. Subsequently, MRCP was acquired that revealed a cystic duct stone causing obstruction and inflammation. MRCP can therefore be a useful tool for clinically and imaging-wise difficult cases. We used HIDA scan for a couple of patients with ultrasound and MRCP findings of acalculous cholecystitis, to confirm the diagnoses. The utility of hepatobiliary scintigraphy has proven extremely useful in the diagnosis of acute cholecystitis, chronic gallbladder disease, biliary leaks, 
biliary obstruction, and biliary atresia.[26] Another important aspect of HIDA scan is to estimate ejection fraction of gallbladder, to exclude an entity called biliary dyskinesia, gallbladder dysmotility or functional gallbladder disorder.[27] Although such patients were not the target population in our study, yet this condition should not be overlooked in patients who present with recurrent right upper quadrant pain mimicking acute cholecystitis,[28] having distended GBs and therefore requiring cholecystectomy.[27] We also found two paediatric acute cholecystitis cases (aged less than 14 years), one with CBD stones and having sickle cell disease (SCD) and other one with congenital anomaly (choledochal cyst-type IB), both with limited and suboptimal ultrasound studies. These patients subsequently had MRCP that showed exquisitely the anatomy of biliary tract and confirmed their diagnoses. Therefore, role of MRCP in children cannot be underestimated in particularly with suspected congenital structural abnormality on initial ultrasounds. Gallstone disease in Sickle Cell Disease SCD is known presentation in children in Saudi Arabia, for which laparoscopic cholecystectomy is advocated as an operative choice.[29-31] Even for adult SCD patients, prophylactic cholecystectomy has been advocated. [32]

Single-center, retrospective analysis and small sample size were considered few of our study limitations. However, we feel that clinical, laboratory and ultrasound findings in patients with acute cholecystitis still need to be emphasized to filter subset of patients that could benefit from MRCP. Availability of MRI facility should not be clinical practice to have this study that requires both time and cost to hospital facilities. Although detection of associated anomalies is not uncommon on MRCP, yet some of these can be found on ultrasound (e.g., choledochal cyst), and even if those cannot be found (e.g., low CBD insertion), these may be identified on careful laparoscopic approach.

For selected or difficult patients, MRCP still remains a good option to delineate biliary anatomy and to diagnose cause of biliary obstruction. Further larger scale studies are needed to define a pathway for adopting MRCP in patients with acute cholecystitis, both in adult and paediatric population, for better patient care and counselling, surgical approach and/ or management options.

\section{CONCLUSIONS}

Magnetic resonance cholangiopancreatography is helpful in identifying causes and anomalies in patients with acute cholecystitis and deranged or obstructive liver tests.

Financial or Other Competing Interests: None.

\section{REFERENCES}

[1] Halpin V. Acute cholecystitis. BMJ Clin Evid 2014;2014:0411.

[2] Bagla P, Sarria JC, Riall TS. Management of acute cholecystitis. Curr Opin Infect Dis 2016;29(5):508-13.

[3] Nasr MM. An Innovative emergency laparoscopic cholecystectomy technique; early results towards complication free surgery. J Gastrointest Surg 2017;21(2):302-11.

[4] Khan SU, Soh JY, Muhibullah N, et al. Emergency laparoscopic cholecystectomy: is dedicated hot gall bladder list cost effective? J Ayub Med Coll Abbottabad 2019;31(1):3-7.

[5] Costi R, Gnocchi A, Di Mario F, et al. Diagnosis and management of choledocholithiasis in the golden age of imaging, endoscopy and laparoscopy. World J Gastroenterol 2014;20(37):13382-401.

[6] Oppenheimer DC, Rubens DJ. Sonography of acute cholecystitis and its mimics. Radiol Clin North Am 2019;57(3):535-48.

[7] Qiu Y, Yang Z, Li Z, et al. Is preoperative MRCP necessary for patients with gallstones? An analysis of the factors related to missed diagnosis of choledocholithiasis by preoperative ultrasound. BMC Gastroenterol 2015;15:158.

[8] Petrescu I, Bratu AM, Petrescu S, et al. CT vs. MRCP in choledocholithiasis jaundice. J Med Life 2015;8(2):22631.

[9] Wong HP, Chiu YL, Shiu BH, et al. Preoperative MRCP to detect choledocholithiasis in acute calculous cholecystitis. J Hepatobiliary Pancreat Sci 2012;19(4):458-64.

[10] Singh A, Mann HS, Thukral CL, et al. Diagnostic accuracy of MRCP as compared to ultrasound/CT in patients with obstructive jaundice. J Clin Diagn Res 2014;8(3):103-7.

[11] Bates DD, LeBedis CA, Soto JA, et al. Use of magnetic resonance in pancreaticobiliary emergencies. Magn Reson Imaging Clin N Am 2016;24(2):433-48.

[12] Chang JH, Lee IS, Lim YS, et al. Role of magnetic resonance cholangiopancreatography for choledocholithiasis: analysis of patients with negative MRCP. Scand J Gastroenterol 2012;47(2):217-24.

[13] Tonolini M, Ravelli A, Villa C, et al. Urgent MRI with MR cholangiopancreatography (MRCP) of acute cholecystitis and related complications: diagnostic role and spectrum of imaging findings. Emerg Radiol 2012;19(4):341-8.

[14] Chen JE, Kadribegic A, Sarkany D. Bilirubin correlation may preclude MRCP in acute cholecystitis patients with normal common bile duct diameter. AJR Am J Roentgenol 2019;212(5):1-6.

[15] Chisholm PR, Patel AH, Law RJ, et al. Preoperative predictors of choledocholithiasis in patients presenting with acute calculous cholecystitis. Gastrointest Endosc 2019;89(5):977-983.e2.

[16] Boys JA, Doorly MG, Zehetner J, et al. Can ultrasound common bile duct diameter predict common bile duct stones in the setting of acute cholecystitis? Am J Surg 2014;207(3):432-5.

[17] Al-Jiffry BO, Elfateh A, Chundrigar T, et al. Non-invasive assessment of choledocholithiasis in patients with gallstones and abnormal liver function. World J Gastroenterol 2013;19(35):5877-82.

[18] Sinha R, Gardner T, Padala K, et al. Double-duct sign in the clinical context. Pancreas 2015;44(6):967-70.

[19] Barut B, Gönültaş F, Gök AFK, et al. Management of acute cholecystitis during pregnancy: a single center experience. Ulus Travma Acil Cerrahi Derg 2019;25(2):154-8. 
[20] Kang SK, Heacock L, Doshi AM, et al. Comparative performance of non-contrast MRI with HASTE vs. contrast-enhanced MRI/3D-MRCP for possible choledocholithiasis in hospitalized patients. Abdom Radiol (NY) 2017;42(6):1650-8.

[21] Tonolini M, Ierardi AM, Patella F, et al. Early crosssectional imaging following open and laparoscopic cholecystectomy: a primer for radiologists. Insights Imaging 2018;9(6):925-41.

[22] Loozen CS, Oor JE, van Ramshorst B, et al. Conservative treatment of acute cholecystitis: a systematic review and pooled analysis. Surg Endosc 2017;31(2):504-15.

[23] Alonso EB, Mata AM, Trastoy PV, et al. Readmissions due to acute biliary edematous pancreatitis in patients without cholecystectomy. Rev Esp Enferm Dig 2016;108(8):473-8.

[24] Fleming CA, Ismail M, Kavanagh RG, et al. Clinical and survival outcomes using percutaneous cholecystostomy tube alone or subsequent interval cholecystectomy to treat acute cholecystitis. J Gastrointest Surg 2020;24(3):627-32.

[25] Hajibandeh S, Popova P, Rehman S. Extended postoperative antibiotics versus no postoperative antibiotics in patients undergoing emergency cholecystectomy for acute calculous cholecystitis: a systematic review and meta-analysis. Surg Innov 2019;26(4):485-96.

[26] Snyder E, Banks KP. Hepatobiliary scintigraphy. StatPearls [Internet]. Treasure Island (FL): StatPearls Publishing 2019.

[27] Eckenrode AH, Ewing JA, Kotrady J, et al. HIDA scan with ejection fraction is over utilized in the management of biliary dyskinesia. Am Surg 2015;81(7):669-73.

[28] Roger J, Heeley T, Graham W, et al. Laparoscopic cholecystectomy for ultrasound normal gallbladders: should we forego hepatobiliary iminodiacetic acid scans? Can J Rural Med 2019;24(2):61-4.

[29] Al Talhi Y, Shirah BH, Altowairqi M, et al. Laparoscopic cholecystectomy for cholelithiasis in children with sickle cell disease. Clin J Gastroenterol 2017;10(4):320-6.

[30] Al-Salem AH, Issa H. Laparoscopic cholecystectomy in children with sickle cell anemia and the role of ERCP. Surg Laparosc Endosc Percutan Tech 2012;22(2):139-42.

[31] Goodwin EF, Partain PI, Lebensburger JD, et al. Elective cholecystectomy reduces morbidity of cholelithiasis in pediatric sickle cell disease. Pediatr Blood Cancer 2017;64(1):113-20.

[32] Muroni M, Loi V, Lionnet F, et al. Prophylactic laparoscopic cholecystectomy in adult sickle cell disease patients with cholelithiasis: a prospective cohort study. Int J Surg 2015;22:62-6. 